\title{
Bounds for Laplacian-type graph energies
}

\author{
Ivan Gutman, Emina Milovanović, and Igor \\ Milovanović
}




\title{
BOUNDS FOR LAPLACIAN-TYPE GRAPH ENERGIES
}

\author{
IVAN GUTMAN, EMINA MILOVANOVIĆ, AND IGOR MILOVANOVIĆ
}

Received 24 February, 2014

\begin{abstract}
Let $G$ be an undirected simple and connected graph with $n$ vertices $(n \geq 3)$ and $m$ edges. Denote by $\mu_{1} \geq \mu_{2} \geq \cdots \geq \mu_{n-1}>\mu_{n}=0, \gamma_{1} \geq \gamma_{2} \geq \cdots \geq \gamma_{n}$, and $\rho_{1} \geq \rho_{2} \geq \cdots \geq$ $\rho_{n-1}>\rho_{n}=0$, respectively, the Laplacian, signless Laplacian, and normalized Laplacian eigenvalues of $G$. The Laplacian energy, signless Laplacian energy, and normalized Laplacian energy of $G$ are defined as $L E=\sum_{i=1}^{n}\left|\mu_{i}-\frac{2 m}{n}\right|, S L E=\sum_{i=1}^{n}\left|\gamma_{i}-\frac{2 m}{n}\right|$, and $N L E=$ $\sum_{i=1}^{n}\left|\rho_{i}-1\right|$, respectively. Lower bounds for $L E, S L E$, and $N L E$ are obtained.
\end{abstract}

2010 Mathematics Subject Classification: 05C50; 05C07

Keywords: energy (of graph), Laplacian energy, signless Laplacian energy, normalized Laplacian energy, Randić energy

\section{INTRODUCTION}

Let $G$ be an undirected simple and connected graph with $n$ vertices $(n \geq 2)$ and $m$ edges, and let $d_{1}, d_{2}, \ldots, d_{n}$ be its vertex degrees.

If the $i$-th and $j$-th vertex of the graph $G$ are adjacent, we write $i \sim j$. Then the adjacency matrix $\mathbf{A}=\left(a_{i j}\right)$ of $G$ is defined as

$$
a_{i j}= \begin{cases}1 & \text { if } i \neq j \text { and } i \sim j \\ 0 & \text { otherwise. }\end{cases}
$$

The eigenvalues $\lambda_{1} \geq \lambda_{2} \geq \cdots \geq \lambda_{n}$ of $\mathbf{A}$ form the (ordinary) spectrum of $G$; for details on the respective spectral theory see [9].

Denote by $\mathbf{D}$ the diagonal matrix of the vertex degrees of $G$. The Laplacian matrix of $G$ is $\mathbf{L}=\mathbf{D}-\mathbf{A}$ and its eigenvalues are $\mu_{1} \geq \mu_{2} \geq \cdots \geq \mu_{n-1}>\mu_{n}=0$ (see $[3,16,25])$. In addition, $\mathbf{Q}=\mathbf{D}+\mathbf{A}$ is the signless Laplacian matrix of $G$ and its eigenvalues will be denoted by $\gamma_{1} \geq \gamma_{2} \geq \cdots \geq \gamma_{n} \geq 0$ [10,11].

Because the graph $G$ is assumed to be connected, it has no isolated vertices (i.e., $d_{i}>0$ for all $\left.1 \leq i \leq n\right)$ and therefore the matrix $\mathbf{D}^{-1 / 2}$ is well-defined. Then $\mathbf{L}^{*}=\mathbf{D}^{-1 / 2} \mathbf{L} \mathbf{D}^{-1 / 2}$ is called the normalized Laplacian matrix of the graph $G$. Its 
eigenvalues are $\rho_{1} \geq \rho_{2} \geq \cdots \geq \rho_{n-1}>\rho_{n}=0$. For details of the spectral theory of the normalized Laplacian matrix see [8].

It is convenient to write the normalized Laplacian matrix as $\mathbf{I}-\mathbf{R}$, where $\mathbf{R}$ is the so-called Randić matrix [4,29,30], whose $(i, j)$-entry is

$$
r_{i j}=\left\{\begin{array}{cl}
1 / \sqrt{d_{i} d_{j}} & \text { if } i \neq j \quad \text { and } i \sim j \\
0 & \text { otherwise. }
\end{array}\right.
$$

The (ordinary) energy of the graph $G$ is defined as [23]

$$
E=E(G)=\sum_{i=1}^{n}\left|\lambda_{i}\right| .
$$

Its theory is nowadays well elaborated [23]. Energy-like spectral invariants have been introduced also for other graph matrices [18]. In this paper we are concerned with the Laplacian [21,23], signless Laplacian [1], and normalized Laplacian (or Randić) energies [5,20], defined as

$$
\begin{array}{r}
L E=L E(G)=\sum_{i=1}^{n}\left|\mu_{i}-\frac{2 m}{n}\right| \\
S L E=S L E(G)=\sum_{i=1}^{n}\left|\gamma_{i}-\frac{2 m}{n}\right| \\
N L E=N L E(G)=\sum_{i=1}^{n}\left|\rho_{i}-1\right|
\end{array}
$$

respectively. In what follows lower bounds for $L E, S L E$ and $N L E$ are obtained.

Remark 1. In analogy to (1.1), the "Randić energy" is defined as the sum of the absolute values of the eigenvalues of the Randić matrix. It has been shown in [20], that the Randić energy coincides with the normalized signless Laplacian energy.

Remark 2. One could also consider the normalized signless Laplacian matrix, $\mathbf{D}^{-1 / 2} \mathbf{Q} \mathbf{D}^{-1 / 2}$ and its "energy" (sum of absolute values of eigenvalues). However, the energy of this matrix is exactly the same as the normalized Laplacian energy, $N L E$ [20]. For the general definition of the energy of a matrix see [28].

The Laplacian, signless Laplacian, and normalized (or Randić) Laplacian spreads of a graph $G$ are defined as $L S(G)=\mu_{1}-\mu_{n-1}, S L S(G)=\gamma_{1}-\gamma_{n}$, and $N L S(G)=\rho_{1}-\rho_{n-1}$, respectively (see $\left.[5,13,15,24]\right)$.

\section{PRELIMINARIES}

In this section we recall some results from spectral graph theory, and state a few analytical inequalities needed for our work. 
Lemma 1 ([3]). Let $G$ be an undirected simple and connected graph with $n, n \geq 2$, vertices and $m$ edges. Then

$$
\sum_{i=1}^{n-1} \mu_{i}=\sum_{i=1}^{n} d_{i}=2 m \quad \text { and } \quad \sum_{i=1}^{n-1} \mu_{i}^{2}=\sum_{i=1}^{n} d_{i}^{2}+\sum_{i=1}^{n} d_{i}=M_{1}+2 m
$$

where $M_{1}$ is the sum of squares of the vertex degrees, usually referred to as the first Zagreb index (see [2,7,19]).

Lemma 2 ([12]). Let $G$ be an undirected simple and connected graph with $n, n \geq$ 2 , vertices and $m$ edges. Then

$$
\frac{M_{1}}{m} \geq 2 \sqrt{\frac{M_{1}}{n}} \geq \frac{4 m}{n} .
$$

Lemma 3 ([31]). Let $G$ an (n,m)-graph, such that $n \geq 3$ and $m \geq 1$. Then

$$
L E(G) \geq \mu_{1}-\mu_{n-1}+\frac{2 m}{n}
$$

with equality if and only if $n=3$ or for $n \geq 4$ if $\mu_{2}=\cdots=\mu_{n-2}=\frac{2 m}{n}$.

Lemma 4 ([26]). Let $G$ be an undirected simple and connected graph with $n, n \geq$ 3 , vertices and $m$ edges. Then

$$
L S(G)=\mu_{1}-\mu_{n-1} \leq \sqrt{\frac{2}{n-1}} \sqrt{(n-1)\left(M_{1}+2 m\right)-4 m^{2}} .
$$

Equality holds if and only if $G \cong K_{n}$.

Lemma 5 ([27]). Let $a_{1}, a_{2}, \ldots, a_{n}$ be real numbers and $p_{1}, p_{2}, \ldots, p_{n}$ non-negative real numbers with the property $p_{1}+p_{2}+\cdots+p_{n}=1$. Then, for each $\alpha, \alpha \leq 0$ and $\alpha \geq 1$,

$$
\sum_{i=1}^{n} p_{i} a_{i}^{\alpha} \geq\left(\sum_{i=1}^{n} p_{i} a_{i}\right)^{\alpha}
$$

For the case $0 \leq \alpha \leq 1$, the opposite inequality is valid. Equality in (2.4) holds if and only if $\alpha=0$ or $\alpha=1$ or $a_{1}=a_{2}=\cdots=a_{n}$.

Lemma 6 ([6]). Let $a_{1}, a_{2}, \ldots, a_{n}$ be real numbers, and assume that there are $r, R \in \mathbb{R}$ such that $-\infty<r \leq a_{i} \leq R<+\infty$, for each $i=1,2, \ldots, n$. Then for any non-negative $p_{1}, p_{2}, \ldots, p_{n}$ with the property $p_{1}+p_{2}+\cdots+p_{n}=1$,

$$
0 \leq \sum_{i=1}^{n} p_{i} a_{i}^{2}-\left(\sum_{i=1}^{n} p_{i} a_{i}\right)^{2} \leq \frac{1}{2}(R-r) \sum_{i=1}^{n} p_{i}\left|a_{i}-\sum_{i=1}^{n} p_{i} a_{i}\right| .
$$

The constant $\frac{1}{2}$ is sharp. 
Lemma 7 ([32]). Let $G$ be an undirected simple and connected graph with $n, n \geq$ 2 , vertices and $m$ edges. Then

$$
\sum_{i=1}^{n-1} \rho_{i}=n \quad \text { and } \quad \sum_{i=1}^{n} \rho_{i}^{2}=n+2 R_{-1}
$$

where $R_{-1}=\sum_{i \sim j} \frac{1}{d_{i} d_{j}}$; for details on the graph invariant $R_{-1}$ see $[4,22]$.

Lemma 8 ([17]). Let $G$ be an undirected simple and connected graph with $n, n \geq$ 2 , vertices and $m$ edges. Then

$$
\sum_{i=1}^{n} \gamma_{i}=\sum_{i=1}^{n} d_{i}=2 m \quad \text { and } \quad \sum_{i=1}^{n} \gamma_{i}^{2}=\sum_{i=1}^{n} d_{i}^{2}+\sum_{i=1}^{n} d_{i}=M_{1}+2 m
$$

where $M_{1}$ is the first Zagreb index.

Lemma 9 ([17]). The signless Laplacian spread has an upper bound

$$
S L S(G) \leq \sqrt{\frac{2\left[n\left(M_{1}+2 m\right)-4 m^{2}\right]}{n}} .
$$

Lemma 10 ([14]). Suppose that $G$ is a graph without isolated vertices. Then

$$
\mu_{1}-\mu_{n-1} \geq \frac{2}{n-1} \sqrt{(n-1)\left(2 m+M_{1}\right)-4 m^{2}} .
$$

\section{MAIN RESUlTS}

3.1. Lower bound for Laplacian energy

Theorem 1. Let $G$ be an undirected connected graph with $n, n \geq 3$, vertices and $m$ edges. Then

$$
L E(G) \geq \frac{2 m}{n}+\frac{2}{n-1} \sqrt{(n-1)\left(2 m+M_{1}\right)-4 m^{2}} .
$$

Proof. Inequality (3.1) directly follows from inequalities (2.2) and (2.7).

Corollary 1. Let $G$ be an undirected graph with $n, n \geq 3$, vertices and $m$ edges. Then

$$
L E(G) \geq \frac{2 m}{n}+\frac{2}{n-1} \sqrt{\frac{2 m(n(n-1)-2 m)}{n}} .
$$

Corollary 2. Let $G$ be an undirected simple and connected $k$-regular graph with $n, n \geq 3$, vertices and $m$ edges, $1<k \leq n-1$. Then

$$
L E(G) \geq k+\frac{2}{n-1} \sqrt{n k(n-k-1)} .
$$


Theorem 2. Let $G$ be an undirected simple and connected graph with $n, n \geq 3$ vertices and $m$ edges. Then

$$
L E(G) \geq \sqrt{\frac{2}{n-1}} \sqrt{(n-1)\left(M_{1}+2 m\right)-4 m^{2}} .
$$

Proof. For $n-1$ and $p_{i}:=\frac{1}{n-1}, a_{i}:=\mu_{i}, i=1,2, \ldots n-1, r:=\mu_{n-1}$ and $R:=\mu_{1}$, the inequality (2.5) transforms into

$$
\frac{1}{n-1} \sum_{i=1}^{n-1} \mu_{i}^{2}-\frac{1}{(n-1)^{2}}\left(\sum_{i=1}^{n-1} \mu_{i}\right)^{2} \leq \frac{\mu_{1}-\mu_{n-1}}{2(n-1)} \sum_{i=1}^{n-1}\left|\mu_{i}-\frac{1}{n-1} \sum_{i=1}^{n-1} \mu_{i}\right|
$$

i.e., based on Lemma 1,

$$
(n-1)\left(M_{1}+2 m\right)-4 m^{2} \leq \frac{n-1}{2}\left(\mu_{1}-\mu_{n-1}\right) \sum_{i=1}^{n-1}\left|\mu_{i}-\frac{2 m}{n-1}\right| .
$$

Since

$$
\sum_{i=1}^{n-1}\left|\mu_{i}-\frac{2 m}{n-1}\right| \leq \sum_{i=1}^{n}\left|\mu_{i}-\frac{2 m}{n}\right|=L E(G)
$$

using inequality (2.3), from the above inequality we obtain (3.2).

Using Lemma 2, we arrive at the following $(n, m)$-type lower bound for the Laplacian energy:

Corollary 3. Let $G$ be an undirected simple and connected graph with $n, n \geq 3$, vertices and $m$ edges. Then

$$
L E(G) \geq \sqrt{\frac{4 m(n(n-1)-2 m)}{n(n-1)}} .
$$

Corollary 4. Let $G$ be an undirected simple and connected $k$-regular graph with $n, n \geq 3$, vertices and $m$ edges, $1<k \leq n-1$. Then

$$
L E(G)>\sqrt{\frac{2 n k(n-k-1)}{n-1}} .
$$

Remark 3. Since for undirected $k$-regular graphs, $L E=E$, the inequality in Corollary 4 provides a lower bound also for the ordinary energy.

Inequalities (3.1) and (3.2) are incomparable. Thus, for example, if $G \cong K_{n}$, then inequality (3.1) is stronger than (3.2), but if $G \cong K_{1, n-1}, n \geq 8$, then the opposite is valid. 


\subsection{Lower bound for signless Laplacian energy}

Theorem 3. Let $G$ be an undirected simple and connected graph with $n, n \geq 3$, vertices and $m$ edges. Then

$$
S L E(G) \geq \sqrt{\frac{2\left(n\left(M_{1}+2 m\right)-4 m^{2}\right)}{n}} .
$$

Proof. For $p_{i}:=\frac{1}{n}, a_{i}=\gamma_{i}, i=1,2, \ldots, n, r=\gamma_{n}$ and $R=\gamma_{1}$, the inequality (2.5) becomes

$$
\frac{1}{n} \sum_{i=1}^{n} \gamma_{i}^{2}-\frac{1}{n^{2}}\left(\sum_{i=1}^{n} \gamma_{i}\right)^{2} \leq \frac{\gamma_{1}-\gamma_{n}}{2 n} \sum_{i=1}^{n}\left|\gamma_{i}-\frac{2 m}{n}\right| .
$$

Bearing in mind Lemma 8 , the above inequality becomes

$$
n\left(M_{1}+2 m\right)-4 m^{2} \leq \frac{n}{2} S L S(G) \times S L E(G) .
$$

By Lemma 9 and the above inequality, we obtain (3.4).

Bearing in mind Lemma 2 and inequality (3.4), we arrive at a lower bound for $S L E(G)$ depending only on the parameter $m$.

Corollary 5. Let $G$ be an undirected simple and connected graph with $n, n \geq 3$, vertices and $m$ edges. Then

$$
\operatorname{SLE}(G) \geq 2 \sqrt{m} .
$$

Corollary 6. Let $G$ be an undirected simple and connected graph with $n, n \geq 3$, vertices and $m$ edges, which is $k$-regular, $1<k \leq n$. Then

$$
\operatorname{SLE}(G) \geq \sqrt{2 n k} .
$$

\subsection{Lower bound for normalized Laplacian energy}

Theorem 4. Let $G$ be an undirected simple and connected graph with $n, n \geq 3$, vertices and $m$ edges. Let, as before, $R_{-1}=\sum_{i \sim j} \frac{1}{d_{i} d_{j}}$. Then

$$
N L S(G) \leq \sqrt{\frac{2}{n-1}} \sqrt{2(n-1) R_{-1}-n} .
$$

Equality holds if and only if $G \cong K_{n}$.

Proof. According to (2.6) we have that

$$
\begin{aligned}
(n-1)\left(n+2 R_{-1}\right)-n^{2} & =(n-1) \sum_{i=1}^{n-1} \rho_{i}^{2}-\left(\sum_{i=1}^{n-1} \rho_{i}\right)^{2} \\
& =\sum_{1 \leq i<j \leq n-1}\left(\rho_{i}-\rho_{j}\right)^{2} .
\end{aligned}
$$


By Lemma 5, i.e., by inequality (2.4), for $n=2$ and $\alpha=2$, we get

$$
\left(\rho_{1}-\rho_{i}\right)^{2}+\left(\rho_{i}-\rho_{n-1}\right)^{2} \geq \frac{1}{2}\left(\rho_{1}-\rho_{n-1}\right)^{2}
$$

for each $i=2,3, \ldots, n-2$. Then,

$$
\begin{aligned}
\sum_{1 \leq i<j \leq n-1}\left(\rho_{i}-\rho_{j}\right)^{2} & \geq \sum_{i=2}^{n-2}\left[\left(\rho_{1}-\rho_{i}\right)^{2}+\left(\rho_{i}-\rho_{n-1}\right)^{2}\right]+\left(\rho_{1}-\rho_{n-1}\right)^{2} \\
& \geq \frac{n-3}{2}\left(\rho_{1}-\rho_{n-1}\right)^{2}+\left(\rho_{1}-\rho_{n-1}\right)^{2} \\
& =\frac{n-1}{2}\left(\rho_{1}-\rho_{n-1}\right)^{2}
\end{aligned}
$$

which combined with (3.6) yields

$$
(n-1)\left(n+2 R_{-1}\right)-n^{2}=2(n-1) R_{-1}-n \geq \frac{n-1}{2}\left(\rho_{1}-\rho_{n-1}\right)^{2}
$$

from which the inequality (3.5) follows.

Equality in (3.7) holds if and only if $\rho_{1}=\rho_{2}=\cdots=\rho_{n-1}$. Therefore, equality in (3.5) holds if and only if $G \cong K_{n}$. This completes the proof of Theorem 4 .

Corollary 7. Let $G$ be an undirected simple nad connected $k$-regular graph, $1<$ $k \leq n-1$, with $n, n \geq 3$, vertices and $m$ edges. Then

$$
N L S(G) \leq \sqrt{\frac{2 n(n-k-1)}{(n-1) k}} .
$$

Equality holds if and only if $k=n-1$, i.e., $G \cong K_{n}$.

We now state a theorem, analogous to Theorem 2, which provides a lower bound for $N L E$ in terms of parameters $n$ and $R_{-1}$.

Theorem 5. Let $G$ be an undirected simple and connected graph with $n, n \geq 3$, vertices and $m$ edges. Then

$$
N L E(G) \geq \sqrt{\frac{2}{n-1}} \sqrt{2(n-1) R_{-1}-n} .
$$

Proof. For $n:=n-1, p_{i}:=\frac{1}{n-1}, a_{i}:=\rho_{i}, i=1,2, \ldots, n-1, r=\rho_{n-1}$ and $R=\rho_{1}$, inequality (2.5) becomes

$$
\frac{1}{n-1} \sum_{i=1}^{n-1} \rho_{i}^{2}-\frac{1}{(n-1)^{2}}\left(\sum_{i=1}^{n-1} \rho_{i}\right)^{2} \leq \frac{\rho_{1}-\rho_{n-1}}{2(n-1)} \sum_{i=1}^{n-1}\left|\rho_{i}-\frac{1}{n-1} \sum_{i=1}^{n-1} \rho_{i}\right| .
$$

Having in mind Lemma 7 , the above inequality transforms into

$$
(n-1)\left(n+2 R_{-1}\right)-n^{2} \leq \frac{n-1}{2} N L S(G) \sum_{i=1}^{n-1}\left|\rho_{i}-\frac{n}{n-1}\right| .
$$


Since

according to (3.9) we obtain

$$
\sum_{i=1}^{n-1}\left|\rho_{i}-\frac{n}{n-1}\right| \leq \sum_{i=1}^{n}\left|\rho_{i}-1\right|
$$

$$
(n-1)\left(n+2 R_{-1}\right)-n^{2} \leq \frac{n-1}{2} N L S(G) N L E(G) .
$$

Combining (3.5) and (3.10) we arrive at (3.8).

Remark 4. For a $k$-regular graph, $R_{-1}=m / k^{2}=n /(2 k)$. Since for $k$-regular graphs, $N L E=\frac{1}{k} E=\frac{1}{k} L E$, inequality (3.8) is equivalent to the result proven in Corollary 4.

\section{REFERENCES}

[1] N. Abreu, D. M. Cardoso, I. Gutman, E. A. Martins, and R. M., "Bounds for the signless Laplacian energy," Linear Algebra Appl., vol. 435, pp. 2365-2374, 2011.

[2] V. Andova, S. Bogoev, D. Dimitrov, M. Pilipczuk, and R. Škrekovski, "On the Zagreb index inequality of graphs with prescribed vertex degrees," Discr. Appl. Math., vol. 159, pp. 852-858, 2011.

[3] N. L. Biggs, Algebraic Graph Theory. Cambridge: Cambridge Univ. Press., 1974.

[4] S. B. Bozkurt, A. D. Güngör, I. Gutman, and A. S. Çevik, "Randić matrix and Randić energy," MATCH Commun. Math. Comput. Chem., vol. 64, pp. 239-250, 2010.

[5] M. Cavers, S. Fallat, and S. Kirkland, "On the normalized Laplacian and general Randić index of graphs," Linear Algebra Appl., vol. 33, pp. 172-190, 2010.

[6] P. Cerone and S. S. Dragomir, "A refinement of the Grüss inequality and applications," Tamkang J. Math., vol. 38, no. 1, pp. 37-49, 2007

[7] S. Chen and W. Liu, "Extremal Zagreb indices of graphs with a given number of cut edges," Graphs Combin., vol. 30, pp. 109-118, 2014.

[8] F. R. K. Chung, Spectral Graph Theory. Providence: Am. Math. Soc., 1997.

[9] D. Cvetković, M. Doob, and H. Sachs, Spectra of Graphs - Theory and Application. New York: Academic Press, 1980.

[10] D. Cvetković, P. Rowlinson, and S. Simi' c, "Signless Laplacian of finite graphs," Linear Algebra Appl., vol. 423, pp. 155-171, 2007.

[11] D. Cvetković and S. Simić, "Towards a spectral theory of graph based on the signless Laplacian ii," Linear Algebra Appl., vol. 432, pp. 2257-2277, 2010.

[12] C. S. Edwards, "The largest vertex degree sum for a triangle in a graph," Bull. London Math. Soc., vol. 9, pp. 203-208, 1977.

[13] Y. Fan, J. Xu, Y. Wang, and D. Liang, "The Laplacian spread of a tree," Discr. Math. Theoret. Comput. Sci., vol. 10, pp. 79-86, 2008.

[14] G. H. Fath-Tabar and A. R. Ashrafi, "Some remarks on Laplacian eigenvalues and Laplacian energy of graphs," Math. Commun., vol. 15, no. 2, pp. 443-451, 2010.

[15] H. Gomes, I. Gutman, E. A. Martins, M. Robbiano, and B. San Martín, "On Randić spread," MATCH Commun. Math. Comput. Chem., vol. 72, pp. 249-266, 2014.

[16] R. Grone and R. Merris, "The Laplacian spectrum of a graph ii," SIAM J. Discrete Math., vol. 7, pp. 221-229, 1994.

[17] A. D. M. Güngör, A. S. Çevik, and N. Habibi, "New bounds for the spread of the signless Laplacian spectrum," Math. Ineq. Appl., in press. 
[18] I. Gutman, "Comparative studies of graph energies," Bull. Acad. Serbe Sci. Arts (Cl. Math. Natur., vol. 144, pp. 1-17, 2012.

[19] I. Gutman and K. C. Das, "The first Zagreb index 30 years after," MATCH Commun. Math. Comput. Chem., vol. 50, pp. 83-92, 2004.

[20] I. Gutman, B. Furtula, and B. Bozkurt, "On Randić energy," Linear Algebra Appl., vol. 442, pp. $50-57,2014$

[21] I. Gutman and B. Zhou, "Laplacian energy of graph," Linear Algebra Appl., vol. 414, pp. 29-37, 2006.

[22] X. Li and I. Gutman, Mathematical Aspects of Randić-Type Molecular Structure Descriptors. Kragujevac: Univ. Kragujevac, 2006.

[23] X. Li, Y. Shi, and I. Gutman, Graph Energy. New York: Springer, 2012.

[24] M. H. Liu and B. L. Liu, "Signless Laplacian spread," Linear Algebra Appl., vol. 309, pp. 2727 2732, 2009.

[25] R. Merris, "Laplacian matrices of graphs: A survey," Linear Algebra Appl., vol. 197-198, pp. 143-176, 1994.

[26] I. v. Milovanović and E. I. Milovanović, "Remark on inequalities for the Laplacian spread of graphs," Czech. Math. J., in press.

[27] D. S. Mitrinović, J. E. Pečari' c, and A. M. Fink, Classical and New Inequalities in Analysis. Dordrecht: Kluwer, 1993.

[28] V. Nikiforov, “The energy of graphs and matrices," J. Math. Anal. Appl., vol. 326, pp. 1472-1475, 2007.

[29] J. A. Rodríguez, “A spectral approach to the Randić index," Linear Algebra Appl., vol. 400, pp. 339-344, 2005.

[30] J. A. Rodríguez and J. M. Sigarreta, "On the Randić index and conditional parameters of a graph," MATCH Commun. Math. Comput. Chem., vol. 54, pp. 403-416, 2005.

[31] B. Zhou and I. Gutman, "Nordhaus-Gaddum type relations for the energy and Laplacian energy of graphs," Bull. Acad. Serbe Sci. Arts (Cl. Math. Natur.), vol. 134, pp. 1-11, 2007.

[32] P. Zumstein, Comparison of spectral methods through the adjacency matrix and the Laplacian of a graph, ser. Th. Diploma. Zurich: ETH Zürich, 2005.

Authors' addresses

Ivan Gutman

University of Kragujevac, Faculty of Science, P. O. Box 60, 34000 Kragujevac, Serbia

E-mail address: gutman@kg.ac.rs

Emina Milovanović

University of Niš, Faculty of Electronics Engineering, A. Medvedeva 14, 18000 Nišs, Serbia

E-mail address: ema@elfak.ni.ac.rs

Igor Milovanović

University of Niš, Faculty of Electronics Engineering, A. Medvedeva 14, 18000 Niš, Serbia

E-mail address: igor@elfak.ni.ac.rs 